\title{
An Obata-type theorem on compact Einstein manifolds with boundary
}

\section{Kazuo Akutagawa ${ }^{1}$}

Received: 6 November 2019 / Accepted: 13 January 2021 / Published online: 3 February 2021

(c) The Author(s) 2021

\begin{abstract}
We show a kind of Obata-type theorem on a compact Einstein $n$-manifold $(W, \bar{g})$ with smooth boundary $\partial W$. Assume that the boundary $\partial W$ is minimal in $(W, \bar{g})$. If $\left(\partial W,\left.\bar{g}\right|_{\partial W}\right)$ is not conformally diffeomorphic to $\left(S^{n-1}, g_{S}\right)$, then for any Einstein metric $\check{g} \in[\bar{g}]$ with the minimal boundary condition, we have that, up to rescaling, $\check{g}=\bar{g}$. Here, $g_{S}$ and $[\bar{g}]$ denote respectively the standard round metric on the $(n-1)$-sphere $S^{n-1}$ and the conformal class of $\bar{g}$. Moreover, if we assume that $\partial W \subset(W, \bar{g})$ is totally geodesic, we also show a Gursky-Han type inequality for the relative Yamabe constant of $(W, \partial W,[\bar{g}])$.
\end{abstract}

Keywords Conformal geometry · Constant scalar curvature - Relative Yamabe constant · Einstein metrics

1991 Mathematics Subject Classication 58E11 · 53C21 · 53A30

\section{Introduction and main results}

In $[14,15]$, Obata has proved the following uniqueness theorem for constant scalar curvature metrics (csc metrics for brevity) on a closed Einstein manifold.

Obata Theorem Let $g$ be an Einstein metric on a closed $n$-manifold $M(n \geq 2)$, and $\check{g} \in[g]$ a csc metric. Then, the following uniqueness result holds:

(1) If $(M,[g])$ is conformally equivalent to $\left(S^{n},\left[g_{S}\right]\right)$, then there exist a homothety $\Phi:\left(S^{n}, g_{S}\right) \rightarrow(M, g)$ and a conformal transformation $\varphi \in \operatorname{Conf}\left(S^{n},\left[g_{S}\right]\right)$ such that $\Phi^{*} \stackrel{\check{g}}{=}=\varphi^{*}\left(\Phi^{*} g\right)$.

(2) If $(M,[g])$ is not conformally equivalent to $\left(S^{n},\left[g_{S}\right]\right)$, then, up to rescaling, $\check{g}=g$.

Here, $[g]$ and $g_{S}=g_{S^{n}}$ denote respectively the conformal class of $g$ and the round metric of constant curvature one on the $n$-sphere $S^{n}$.

Supported in part by the Grants-in-Aid for Scientific Research (B), Japan Society for the Promotion of Science, No. $18 \mathrm{H} 01117$.

$凶 \quad$ Kazuo Akutagawa

akutagawa@math.chuo-u.ac.jp

1 Department of Mathematics, Chuo University, Tokyo 112-8551, Japan 
We will first review briefly a uniqueness problem on compact manifolds with boundary, which is related to Obata Theorem. Let $W$ be a compact $n$-manifold $(n \geq 2)$ with non-empty smooth boundary $\partial W$. Let $\bar{g}$ be a metric on $W$ such that $\partial W$ is minimal in $(W, \bar{g})$. We shall call such $\bar{g}$ a relative metric on $W$ (cf. [1]). For the conformal class $[\bar{g}]$, its subset

$$
\begin{aligned}
{[\bar{g}]_{0} } & :=\left\{\tilde{g}=e^{2 f} \cdot \bar{g} \mid f \in C^{\infty}(W), \quad H_{\tilde{g}}=0 \text { on } \partial W\right\} \\
& =\left\{\widetilde{g}=e^{2 f} \cdot \bar{g} \mid f \in C^{\infty}(W), \quad v(f)=0 \text { along } \partial W\right\}
\end{aligned}
$$

is called the relative conformal class of $\bar{g}$, where $H_{\tilde{g}}$ denotes the mean curvature $\partial W$ in $(W, \widetilde{g})$, and $v=v_{\bar{g}}$ denotes the inner unit normal vector field along $\partial W$ with respect to $\bar{g}$. Set

$$
\begin{aligned}
& S_{+}^{n}:=\left\{x=\left(x^{1}, \cdots, x^{n}, x^{n+1}\right) \in S^{n} \subset \mathbb{R}^{n+1} \mid x^{n+1} \geq 0\right\}, \quad S^{n-1}:=\left\{x \in S^{n} \mid x^{n+1}=0\right\}, \\
& \operatorname{Conf}\left(S_{+}^{n},\left[g_{S}\right]\right):=\left\{\varphi \in \operatorname{Conf}\left(S^{n},\left[g_{S}\right]\right) \mid \varphi\left(S_{+}^{n}\right)=S_{+}^{n}\right\},
\end{aligned}
$$

where $g_{S}$ denotes both the round metric on $S^{n}$ and its restriction to $S_{+}^{n}$. Then, we consider the following naive question:

Problem Let $\bar{g}$ be a relative Einstein metric on a compact $n$-manifold $W$ with boundary $\partial W$. Then, for any relative csc metric $\check{g} \in[\bar{g}]_{0}$, the question is whether the following uniqueness holds or not.

(1) If $(W,[\bar{g}])$ is conformally equivalent to $\left(S_{+}^{n},\left[g_{S}\right]\right)$, then there exist a homothety

$\Phi:\left(S_{+}^{n}, g_{S}\right) \rightarrow(W, \bar{g})$ and a conformal transformation $\varphi \in \operatorname{Conf}\left(S_{+}^{n},\left[g_{S}\right]\right)$ such that $\Phi^{*} \stackrel{\check{g}}{=} \varphi^{*}\left(\Phi^{*} g\right)$.

(2) If $(W,[\bar{g}])$ is not conformally equivalent to $\left(S_{+}^{n},\left[g_{S}\right]\right)$, then, up to rescaling, $\check{g}=\bar{g}$.

Like the case of non-positive csc metrics on a closed manifold, if $\bar{g}$ has non-positive scalar curvature $R_{\bar{g}} \leq 0$, then the uniqueness for relative csc metrics in $[\bar{g}]_{0}$ holds, up to rescaling (see the argument of [11, Proof of Theorem 3.6] for proof). Hence, in the above problem, it is enough to consider only the case of positive scalar curvature $R_{\bar{g}}>0$. Unfortunately, there exists a counterexample to the second assertion (2) (see Escobar [7, p. 875] for instance). However, if we assume that $\partial W$ is totally geodesic in $(W, \bar{g})$, then the following holds, which is a relative version of Obata Theorem.

Escobar Theorem ([7, Theorems 3.2, 4.1]). Let $\bar{g}$ be an Einstein metric of positive scalar curvature (positive Einstein metric for brevity) on a compact $n$-manifold $W$ with totally geodesic boundary $\partial W$. Then, for any relative csc metric $\check{g} \in[\bar{g}]_{0}$, the same uniqueness assertions as those (1), (2) in Problem hold.

The proof of Obata Theorem consists of two steps. The first step is to prove that $\check{g} \in[g]$ is also an Einstein metric on $M$. So ignoring the first step, we may regard Obata Theorem as the uniqueness result for Einstein metrics in a given conformal class.

Our main result of this paper is the following, which is released from the assumption that $\partial W$ is totally geodesic in $(W, \bar{g})$.

Theorem 1.1 Let $\bar{g}$ be a relative positive Einstein metric on a compact $n$-manifold $W$ with boundary. Then, for any relative Einstein metric $\stackrel{g}{g} \in[\bar{g}]_{0}$, the following holds:

(1) Assume that $\bar{g}$ is a metric of positive constant curvature, and set $g:=\left.\bar{g}\right|_{\partial W}$.

(1.1) If $(\partial W,[g])$ is conformally equivalent to $\left(S^{n-1},\left[g_{S^{n-1}}\right]\right)$, then there exist a homothety $\Phi:\left(S_{+}^{n}, g_{S}\right) \rightarrow(W, \bar{g})$ and a conformal transformation $\varphi \in \operatorname{Conf}\left(S_{+}^{n},\left[g_{S}\right]\right)$ such that $\Phi^{*} \check{g}=\varphi^{*}\left(\Phi^{*} g\right)$. 
(1.2) If $(\partial W,[g])$ is not conformally equivalent to $\left(S^{n-1},\left[g_{S^{n-1}}\right]\right)$, then, up to rescaling, $\check{g}=\bar{g}$.

(2) If $\bar{g}$ is not a metric of positive constant curvature, then, up to rescaling, $\check{g}=\bar{g}$.

Moreover, if we assume that $\partial W$ is totally geodesic, we also show the following GurskyHan type inequality (cf. [12, Theorem 2.4]):

Theorem 1.2 Under the same setting as Escobar Theorem, the following inequality holds:

$$
Y(W, \partial W,[\bar{g}]) \geq \frac{n}{n-2} \cdot \frac{\operatorname{Vol}(W, \bar{g})^{2 / n}}{\operatorname{Vol}(\partial W, g)^{2 /(n-1)}} Y(\partial W,[g]),
$$

where $g:=\left.\bar{g}\right|_{\partial W}$, and that $Y(W, \partial W,[\bar{g}])$ and $Y(\partial W,[g])$ denote respectively the relative Yamabe constant of $(W, \partial W,[\bar{g}])$ (see Sect. 2 for definition) and the Yamabe constant of $(\partial W,[g])$. Moreover, the equality in (1) holds if and only if $g$ is a Yamabe metric on $\partial W$. When this equality holds, a reverse inequality of (1) holds:

$$
Y(W, \partial W,[\bar{g}]) \leq\left(\frac{\operatorname{Vol}\left(S^{n-1}, g_{S^{n-1}}\right)}{\operatorname{Vol}(\partial W, g)}\right)^{\frac{2}{n(n-1)}} \cdot \frac{Y\left(S_{+}^{n}, S^{n-1},\left[g_{S^{n}}\right]\right)}{Y\left(S^{n-1},\left[g_{S^{n-1}}\right]\right)} Y(\partial W,[g]) .
$$

The equality in (2) also holds if and only if $(W, \bar{g})$ is homothetic to $\left(S_{+}^{n}, g_{S^{n}}\right)$.

This paper is organized as follows. In Sect.2, we recall some background materials, particularly a variational characterization of relative Einstein metrics and the Yamabe problem on compact manifolds with boundary. We also give another counterexample to the second assertion (2) of Problem, which is different from the one in Escobar [7, p. 875]. In Sect. 3, we prove Theorem 1.2. In Sect. 4, we finally prove Theorem 1.1.

\section{Preliminaries}

We first recall to the variational characterization of Einstein metrics on a closed manifold. Let $M$ be a closed $n$-manifold ( $n \geq 3$ ). It is well known that a Riemannian metric on a closed manifold $M$ is Einstein if and only if it is a critical point of the normalized Einstein-Hilbert functional $\mathcal{E}$ on the space $\mathcal{M}(M)$ of all Riemannian metrics on $M$

$$
\mathcal{E}: \mathcal{M}(M) \rightarrow \mathbb{R}, \quad g \mapsto \mathcal{E}(g):=\frac{\int_{M} R_{g} d \mu_{g}}{\operatorname{Vol}(M, g)^{(n-2) / n}},
$$

where $R_{g}, d \mu_{g}$ and $\operatorname{Vol}(M, g)$ denote respectively the scalar curvature and volume measure of $g$, and the volume of $(M, g)$. However, if we consider the analogue of the case of the functional $\mathcal{E}$ on a compact $n$-manifold $W$ with non-empty boundary $\partial W$, then the set of critical points of $\mathcal{E}$ on the space $\mathcal{M}(W)$ is empty (see Proposition 2.1 below), where $\mathcal{M}(W)$ denotes the space of all Riemannian metrics on $W$. In this case, we need to fix a kind of boundary condition for all metrics, and then $\mathcal{E}$ must be restricted to a subspace of $\mathcal{M}(W)$.

For a fixed conformal class $C \in \mathcal{C}(\partial W)$ on $\partial W$, set several subspaces of $\mathcal{M}(W)$ for a as below:

$$
\begin{aligned}
& \mathcal{M}(W, C):=\left\{\bar{g} \in \mathcal{M}(W) \mid\left[\left.\bar{g}\right|_{\partial W}\right]=C\right\}, \\
& \mathcal{M}_{\text {const }}(W, C):=\left\{\bar{g} \in \mathcal{M}(W, C) \mid \exists c \in \mathbb{R} \text { s.t. } H_{\bar{g}}=c \text { on } \partial W\right\}, \\
& \mathcal{M}_{0}(W):=\left\{\bar{g} \in \mathcal{M}(W) \mid H_{\bar{g}}=0 \text { on } \partial W\right\}, \\
& \mathcal{M}_{0}(W, C):=\mathcal{M}(W, C) \cap \mathcal{M}_{0}(W)=\left\{\bar{g} \in \mathcal{M}(W, C) \mid H_{\bar{g}}=0 \text { on } \partial W\right\} .
\end{aligned}
$$

By Proposition 2.1 below, it is reasonable to restrict the functional $\mathcal{E}$ to the subspace either $\mathcal{M}_{0}(W, C)$ or $\mathcal{M}_{0}(W)$. 
Proposition 2.1 Let $W$ be a compact $n$-manifold with boundary and $\mathcal{E}$ the normalized Einstein-Hilbert functional on the space $\mathcal{M}(W)$. Then, the following holds:

(1) $\bar{g} \in \operatorname{Crit}\left(\left.\mathcal{E}\right|_{\mathcal{M}_{0}(W, C)}\right)$ if and only if $\bar{g}$ is a relative Einstein metric with $\left[\left.\bar{g}\right|_{\partial W}\right]=C$.

(2) $\bar{g} \in \operatorname{Crit}\left(\left.\mathcal{E}\right|_{\mathcal{M}_{0}(W)}\right)$ if and only if $\bar{g}$ is an Einstein metric such that $\partial W$ is totally geodesic in $(W, \bar{g})$.

(3) $\operatorname{Crit}(\mathcal{E})=\emptyset, \quad \operatorname{Crit}\left(\left.\mathcal{E}\right|_{\mathcal{M}(W, C)}\right)=\emptyset, \quad \operatorname{Crit}\left(\left.\mathcal{E}\right|_{\mathcal{M}_{\text {const }}(W, C)}\right)=\emptyset$.

Here, for instance, $\operatorname{Crit}(\mathcal{E})$ and $\operatorname{Crit}\left(\left.\mathcal{E}\right|_{\mathcal{M}_{0}(W, C)}\right)$ denote respectively the set of all critical metrics of $\mathcal{E}$ and the set of those of its restriction to $\mathcal{M}_{0}(W, C)$.

Proof Let $\{\bar{g}(t)\}_{t \in(-\varepsilon . \varepsilon)}$ be a smooth family of metrics in $\mathcal{M}(W)$ satisfying $\bar{g}(0)=\bar{g}$. Then, we get the following first variation of $\mathcal{E}$ ([1, Claim 3.1], see also [2, Sect. 2]):

$$
\begin{array}{r}
\left.\frac{d}{d t}\right|_{t=0} \int_{W} R_{\bar{g}(t)} d \mu_{\bar{g}(t)}=-\int_{W}\left\langle\operatorname{Ric}_{\bar{g}}-\frac{1}{2} R_{\bar{g}} \cdot \bar{g}, h\right\rangle_{\bar{g}} d \mu_{\bar{g}} \\
-\int_{\partial W}\left\{2\left(\left.\frac{\partial}{\partial t}\right|_{t=0} H_{\bar{g}(t)}\right)+\left\langle A_{\bar{g}}, h\right\rangle_{\bar{g}}\right\} d \sigma_{\bar{g}},
\end{array}
$$

where $h, \operatorname{Ric}_{\bar{g}}$ and $A_{\bar{g}}$ denote respectively the variational vector $\left.\frac{\partial}{\partial t} \bar{g}(t)\right|_{t=0}$ of $\{\bar{g}(t)\}$, the Ricci curvature of $\bar{g}$ and the second fundamental form of $\partial W$ in $(W, \bar{g})$, and also $d \sigma_{\bar{g}}:=$ $d \mu_{\bar{g}}\lfloor(\partial W)$.

By considering the case that $\{\bar{g}(t)\}_{t \in(-\varepsilon . \varepsilon)} \subset \mathcal{M}_{0}(W)$ with $h=0$ on $\partial W$, one can check that any $\bar{g} \in \operatorname{Crit}(\mathcal{E})$ satisfies $\operatorname{Ric}_{\bar{g}}=\lambda \cdot \bar{g}$ (for $\exists \lambda \in \mathbb{R}$ ). Here, we use that $H_{\bar{g}(t)}=0$ for $t \in(-\varepsilon, \varepsilon)$. By considering the case that $\{\bar{g}(t)\}_{t \in(-\varepsilon . \varepsilon)} \subset \mathcal{M}_{0}(W)$ without any extra condition, one can also get that $A_{\bar{g}}=0$ on $\partial W$. However, since, for any fixed $\bar{g} \in \mathcal{M}(W)$ with $\operatorname{Ric}_{\bar{g}}=\lambda \cdot \bar{g}$ and $A_{\bar{g}}=0$, but $\left.\frac{\partial}{\partial t}\right|_{t=0} H_{\bar{g}(t)}$ is not zero generally for $\{\bar{g}(t)\}_{t \in(-\varepsilon . \varepsilon)} \subset \mathcal{M}(W)$, and hence $\operatorname{Crit}(\mathcal{E})=\emptyset$. Similarly, we get

$\operatorname{Crit}\left(\left.\mathcal{E}\right|_{\mathcal{M}(W, C)}\right), \operatorname{Crit}\left(\left.\mathcal{E}\right|_{\mathcal{M}_{\text {const }}(W, C)}\right) \subset\left\{\bar{g} \in \mathcal{M}(W) \mid \bar{g}\right.$ is Einstein and $H_{\bar{g}}=0$ on $\left.\partial W\right\}$, and hence these are empty.

Assume that $\{\bar{g}(t)\}_{t \in(-\varepsilon . \varepsilon)} \subset \mathcal{M}_{0}(W)$. By the same reason as above, we get the second assertion (2). Assume also that $\{\bar{g}(t)\}_{t \in(-\varepsilon . \varepsilon)} \subset \mathcal{M}_{0}(W, C)$. Then, we get similarly the first assertion (1).

For $\bar{g} \in \mathcal{M}(W), \tilde{g}=e^{2 f} \cdot \bar{g}$, the following holds (cf. [8, (1.4)]):

$$
H_{\tilde{g}}=e^{-f}\left(H_{\bar{g}}+v_{\bar{g}}(f)\right) .
$$

From this, note that $[\bar{g}]_{0} \neq \emptyset$ for any $\bar{g} \in \mathcal{M}(W)$. From now on, we throughout assume that $\bar{g} \in \mathcal{M}_{0}(W)$, namely a relative metric on $W$. The restriction of $\mathcal{E}$ to any relative conformal class $[\bar{g}]_{0}$ is always bounded from below similarly to the case of closed manifolds. Hence, we can consider the following conformal invariant of $(W,[\bar{g}])$

$$
Y(W, \partial W,[\bar{g}]):=\inf _{\tilde{g} \in[\bar{g}]_{0}} \mathcal{E}(\widetilde{g}),
$$

which is called the relative Yamabe constant of (W, $\partial W,[\bar{g}])$, or $(W,[\bar{g}])$ simply (cf. [1]). Then, the following Aubin-type inequality for relative Yamabe constants, the so-called relative Aubin's inequality holds (cf. [8, (4)]):

$$
Y(W, \partial W,[\bar{g}]) \leq Y\left(S_{+}^{n}, S^{n-1},\left[g_{S}\right]\right)
$$

for any compact Riemannian $n$-manifold $(W, \bar{g})$ with minimal boundary $\partial W$. 
The relative Yamabe constant is related to the Yamabe problem on a compact $n$-manifold $W$ with boundary below

The Relative Yamabe Problem For any $\bar{g} \in \mathcal{M}_{0}(W)$, find a minimizer $\check{g} \in[\bar{g}]_{0}$ of $\left.\mathcal{E}\right|_{[\bar{g}]_{0}}$, namely

$$
\mathcal{E}(\check{g})=\inf _{\widetilde{g} \in[\bar{g}]_{0}} \mathcal{E}(\widetilde{g})(=Y(W, \partial W,[\bar{g}])) .
$$

If such a $\check{g}$ exists, it is called a relative Yamabe metric in $[\bar{g}]_{0}$, which is a csc metric with $R_{\check{g}}=Y(W, \partial W,[\bar{g}]) \cdot \operatorname{Vol}(W, \check{g})^{-2 / n}$.

On the relative Yamabe problem, although the formulation (3) in Escobar [8] is slightly different from the above, but these are same each other. The relative Yamabe problem was solved by Cherrier [5] and Escobar [8] under some restrictions. Indeed, Cherrier proved the existence of a minimizer for the Yamabe functional $\left.\mathcal{E}\right|_{[\bar{g}]_{0}}$ provided

$$
Y(W, \partial W,[\bar{g}])<Y\left(S_{+}^{n}, S^{n-1},\left[g_{S}\right]\right) .
$$

Escobar also solved the relative Yamabe problem under one of the restrictions we list below. Here is the list of conditions given in [8, Theorem 6.1] (on further developments, see [4] for instance):

(i) $n=3$, 4, or 5 ,

(ii) $W$ has a non totally geodesic point on $\partial W$,

(iii) $\partial W$ is totally geodesic and $(W, \bar{g})$ is locally conformally flat,

(iv) $\partial W$ is totally geodesic, $n \geq 6$ and the Weyl tensor does not vanish identically on $\partial W$.

Note that, in the case of either (ii) or (iv), the above strict inequality (3) holds. By the above results, it is easy to see that the relative Yamabe problem is generically solvable.

Next, we will give some examples for Escobar Theorem.

Example 2.2 (1) Let $\bar{g}$ be the standard product Einstein metric on $W:=S^{2}(1) \times S_{+}^{2}(1)$, where $S^{2}(1)$ denotes the round 2 -sphere of radius 1 in $\mathbb{R}^{3}$. It is obvious that $\partial W$ is totally geodesic. By Escobar Theorem, this metric $\bar{g}$ is a unique relative csc metric in $[\bar{g}]_{0}$, up to rescaling.

(2) Set $W:=\mathbb{C P}^{2}-B^{4}$. Let $g_{P}$ be the Page metric on $\mathbb{C P}^{2} \#\left(-\mathbb{C P}^{2}\right)=W \sqcup_{\partial W}(-W)$, which is an Einstein metric and not a metric of constant curvature. Then, there exists a natural isometric involution $\iota$ on $\mathbb{C P}^{2} \#\left(-\mathbb{C P}^{2}\right)$ such that the fixed point set of $\iota$ is equal to $\partial W$ (cf. [3, Proof of Theorem 9.125]). Hence, $\partial W$ is totally geodesic. By Escobar Theorem again, the restriction $\left.g_{P}\right|_{W}$ of $g_{P}$ to $W$ is a unique relative csc metric in $\left[\left.g_{P}\right|_{W}\right]_{0}$, up to rescaling.

We now give another counterexample to the second assertion(2) in Problem of Sect. 1, which is different from the one in [7, p. 875].

Counterexample Consider the Clifford torus $\Phi\left(T^{2}\right)$ :

$$
\Phi: T^{2}=S^{1} \times S^{1} \rightarrow S^{3}(1) \subset \mathbb{C}^{2}, \quad(\theta, \phi) \mapsto \frac{1}{\sqrt{2}}\left(e^{\sqrt{-1} \theta}, e^{\sqrt{-1} \phi}\right) \quad(0 \leq \theta, \phi \leq 2 \pi) .
$$

It is a minimal flat torus. Set

$$
V_{1} \sqcup V_{2}=S^{3}(1)-\Phi\left(T^{2}\right) .
$$

It is well known that $\operatorname{Vol}\left(\bar{V}_{1}\right)=\operatorname{Vol}\left(\bar{V}_{2}\right)$, each of which $\bar{V}_{i}(i=1,2)$ is a solid torus with minimal boundary $\partial \bar{V}_{i}=\Phi\left(T^{2}\right)$. Hence, $\bar{g}:=\left.g_{S}\right|_{\bar{V}_{1}}$ is a metric of constant curvature one 
on $\bar{V}_{1}$, and thus it is a relative Einstein metric. However, since $\Phi\left(T^{2}\right)$ is not totally geodesic in $\bar{V}_{1}$, it is follows from Escobar's result [8, Theorem 4.1] that

$$
Y\left(\bar{V}_{1}, \partial \bar{V}_{1},[\bar{g}]\right)<Y\left(S_{+}^{3}, S^{2},\left[g_{S}\right]\right) .
$$

Hence, from Cherrier's result, there exists a relative Yamabe metric $\check{g} \in[\bar{g}]_{0}$ such that $\mathcal{E}(\check{g})=Y\left(\bar{V}_{1}, \partial \bar{V}_{1},[\bar{g}]\right)$. From the fact that $\mathcal{E}(\bar{g})=Y\left(S_{+}^{3}, S^{2},\left[g_{S}\right]\right)>\mathcal{E}(\check{g})$, then $\check{g} \neq \bar{g}$, and thus the uniqueness assertion for relative csc metrics does not hold. Note also that, from Theorem 1.1,(1.2), $\check{g}$ is not an Einstein metric. More generally, any separating embedded minimal hypersurface $N$ in $S^{n}(1)(n \geq 3)$ which is not totally geodesic produces a counterexample as below. Let

$$
W_{1} \sqcup W_{2}=S^{n}(1)-N \text { with } \operatorname{Vol}\left(\bar{W}_{1}\right) \geq \operatorname{Vol}\left(\bar{W}_{2}\right),
$$

and then $\mathcal{E}\left(\left.g_{S^{n}}\right|_{W_{1}}\right) \geq Y\left(S_{+}^{n}, S^{n-1},\left[g_{S^{n}}\right]\right)$. By the same reason as the above, we get the counterexample $\left(\bar{W}_{1},\left.g_{S^{n}}\right|_{\bar{W}_{1}}\right)$ to the second assertion (2) in Problem.

We finally prepare a necessary result for our proof of Theorem 1.1. The following result follows from Frankel-Petersen-Wilhelm theorem [16, Theorem 3] (cf. [9]) for minimal hypersurfaces in a Riemannian manifold with positive Ricci curvature.

Proposition 2.3 Let $(W, \bar{g})$ be a compact Riemannian manifold of positive Ricci curvature with non-empty boundary $\partial W$. Assume that $\partial W$ is a minimal hypersurface. Then, $\partial W$ is connected.

\section{Proofs of Theorem 1.2}

In order to prove Theorem 1.2, we first give another proof of Escobar Theorem, which is slightly different from the one given in Escobar [7, Proof of Theorem 4.1].

Proof of Escobar Theorem. Step 1 For a sufficiently small $\varepsilon>0$, we consider the $\varepsilon$-tubular neighborhood $U_{\varepsilon}(\partial W)$ of $\partial W$ :

$$
U_{\varepsilon}(\partial W)=\left\{p \in W \mid \operatorname{dist}_{\bar{g}}(p, \partial W)<\varepsilon\right\} \cong[0, \varepsilon) \times \partial W .
$$

In terms of Fermi coordinates $\left(t=x^{0}, x\right)=\left(x^{0}, x^{1}, \cdots, x^{n-1}\right)=\exp _{x}(t \cdot v(x))$, the metric $\bar{g}$ can be expressed by

$$
\begin{aligned}
& \bar{g}(t, x)_{\alpha \beta} d x^{\alpha} d x^{\beta} \\
& \quad=d t^{2}+\left\{g(x)_{i j}-2 t A_{\bar{g}}(x)_{i j}+t^{2}\left(-R_{\bar{g}}(0, x)_{0 i 0 j}+\left(A_{\bar{g}} \circ A_{\bar{g}}\right)(x)_{i j}\right)+T(t, x)_{i j}\right\} d x^{i} d x^{j},
\end{aligned}
$$

where $g(x):=\bar{g}(0, x)$ on $\partial W,\left(R_{\bar{g}}\right)_{\alpha \beta \gamma \delta}$ denotes components of the Riemannian curvature tensor of $\bar{g}$, and $T(t, x)=O\left(t^{3}\right)$ as $t \searrow 0$. We use here the summation convention with Roman indices in the range $1 \leq i, j, \cdots \leq n-1$ and Greek in $0 \leq \alpha, \beta, \cdots \leq n-1$. Let $\mathscr{D}(W):=W \sqcup_{\partial W}(-W)$ be the double of $W$. Note that $\mathscr{D}(W)$ has a natural differentiable structure coming from the one of $W$.

Step 2 The metric $\bar{g}$ on $W$ can be extended naturally to a metric $\widehat{g}$ on $\mathscr{D}(W)$. In particular, $\widehat{g}$ can be expressed on the $\varepsilon$-tubular neighborhood $\mathcal{U}_{\varepsilon}(\partial W) \cong(-\varepsilon, \varepsilon) \times \partial W$ of $\partial W$ in $\mathscr{D}(W)$ as

$$
\widehat{g}(t, x)=\left\{\begin{array}{cc}
\bar{g}(t, x) & \text { for }(t, x) \in[0, \varepsilon) \times \partial W, \\
\bar{g}(-t, x) & \text { for }(t, x) \in(-\varepsilon, 0] \times \partial W
\end{array}\right.
$$


For a general relative metric $\bar{g}$ on $W, \widehat{g}$ is only a $C^{0,1}$ metric on $\mathscr{D}(W)$ since $A_{\bar{g}}$ does not vanish generally. But, by the assumption that $\partial W$ is totally geodesic (i.e., $A_{\bar{g}}=0$ on $\partial W$ ), $\widehat{g}$ is a $C^{2,1}$ metric on $\mathscr{D}(W)$. Note also that there exists a natural isometric involution of $\mathscr{D}(W)$ such that $\iota(t, x)=(-t, x)$ on $\mathcal{U}_{\varepsilon}(\partial W) \cong(-\varepsilon, \varepsilon) \times \partial W$.

Step 3 Cover $\mathcal{U}_{\varepsilon}(\partial W)$ by a family of harmonic coordinates. Then, applying DeTurckKazdan's regularity theorem [6] to the relative Einstein $C^{2,1}$ metric $\widehat{g}$ on $\mathscr{D}(W)$, we conclude that $(\mathscr{D}(W), \widehat{g})$ is a real analytic Einstein manifold. Set $\check{g}=u^{4 /(n-2)} \cdot \bar{g}$ on $W$ for $u \in C_{>0}^{\infty}(W)$. Then $u$ satisfies the following:

$$
-\frac{4(n-1)}{n-2} \Delta_{\bar{g}} u+R_{\bar{g}} u=R_{\check{g}} u^{\frac{n+2}{n-2}} \text { on } W .
$$

$u$ can be naturally extended to a positive function $\widehat{u}$ on $\mathscr{D}(W)$, similarly to $\widehat{g}$. In particular, $\widehat{u}(t, x)=u(-t, x)$ on $(-\varepsilon, 0] \times \partial W$. Note that $\frac{\partial u}{\partial t}=0$ on $\partial W$ since $\check{g}$ is also relative metric. Then, $\widehat{u} \in C_{>0}^{1,1}(\mathscr{D}(W))$, and hence, by the elliptic regularity, $\widehat{u} \in C_{>0}^{\infty}(\mathscr{D}(W))$. Setting $\widetilde{g}:=\widehat{u}^{4 /(n-2)} \cdot \widehat{g}$ on $\mathscr{D}(W)$, we get that $\widetilde{g}$ is a $\csc C^{\infty}$ metric on $\mathscr{D}(W)$.

Step 4 Consider the two conformal metrics $\widehat{g}$ and $\widetilde{g}$ on $\mathscr{D}(W)$. By the first step of the proof of Obata Theorem (cf. [17, Proof of Proposition 1.4]), $\widetilde{g}$ is also an Einstein metric, and that it satisfies the following Hessian equation:

$$
\widehat{\nabla}^{2} \phi=\frac{\Delta_{\widehat{g}} \phi}{n} \widehat{g} \quad\left(\phi:=\widehat{u}^{-\frac{2}{n-2}}\right),
$$

where $\widehat{\nabla}$ denotes the Levi-Civita connection of $\widehat{g}$. If $\phi$ is not constant on $\mathscr{D}(W)$, it is known that $(\mathscr{D}(W), \widehat{g})$ is homothetic to $\left(S^{n}, g_{S}\right)$ [14], [18] (cf. [13, Theorem 24]). By the existence of the isometric involution $\iota$ of $(\mathscr{D}(W), \widehat{g})$, the following assertions are equivalent:

- $(\mathscr{D}(W),[\widehat{g}])$ is conformally equivalent to $\left(S^{n},\left[g_{S}\right]\right)$.

- $(W,[\bar{g}])$ is conformally equivalent to $\left(S_{+}^{n},\left[g_{S}\right]\right)$.

By the fact that $\iota$ is also an isometric involution of $(\mathscr{D}(W), \widetilde{g})$, Escobar Theorem now follows from Obata Theorem.

Proof of Theorem 1.2 Set $g:=\left.\bar{g}\right|_{\partial W}$. Since $\bar{g}$ is Einstein, then the Gauss equation for the hypersurface $\partial W$ implies that

$$
R_{g}=\frac{n-2}{n} R_{\bar{g}} \equiv \text { const }>0 \text { on } \partial W .
$$

Moreover, by Escobar Theorem, $\bar{g}$ is a relative Yamabe metric on $W$. Then,

$$
\begin{aligned}
Y(\partial W,[g]) & \leq\left(\int_{\partial W} R_{g} d \mu_{g}\right) \cdot \operatorname{Vol}(\partial W, g)^{-\frac{n-3}{n-1}} \\
& =\frac{n-2}{n}\left(\int_{\partial W} R_{\bar{g}} d \mu_{g}\right) \cdot \operatorname{Vol}(\partial W, g)^{-\frac{n-3}{n-1}} \\
& =\frac{n-2}{n} Y(W, \partial W,[\bar{g}]) \cdot \operatorname{Vol}(W, \bar{g})^{-\frac{2}{n}} \cdot \operatorname{Vol}(\partial W, g)^{\frac{2}{n-1}},
\end{aligned}
$$

and hence we get the desired inequality(1). It is obvious that the equality in the above inequality holds if and only if $g$ is a Yamabe metric on $\partial W$.

Now, we assume that the equality in (1) holds, equivalently that $g$ is a Yamabe metric on $\partial W$. Without loss of generality, we may also assume that $\operatorname{Ric}_{\bar{g}}=(n-1) \bar{g}$. By Gromov's isoperimetric inequality [10] for $(W, \bar{g})$ in $(\mathscr{D}(W), \widehat{g})$, we then have that

$$
\frac{\operatorname{Vol}(\partial W, g)}{\operatorname{Vol}(W, \bar{g})} \geq \frac{\operatorname{Vol}\left(S^{n-1}, g_{S^{n-1}}\right)}{\operatorname{Vol}\left(S_{+}^{n}, g_{S^{n}}\right)}
$$


Moreover, the equality in (5) holds if and only if $(W, \bar{g})$ is isometric to $\left(S_{+}^{n}, g_{S^{n}}\right)$. Combining the inequality (5) with the equality in (1), we obtain the reverse inequality (2) as below:

$$
\begin{aligned}
& Y(W, \partial W,[\bar{g}])=\frac{n}{n-2} \cdot \frac{\operatorname{Vol}(W, \bar{g})^{\frac{2}{n}}}{\operatorname{Vol}(\partial W, g)^{\frac{2}{(n-1)}}} \cdot Y(\partial W,[g]) \\
& =\frac{n(n-1)}{(n-1)(n-2)} \cdot\left(\frac{\operatorname{Vol}(W, \bar{g})}{\operatorname{Vol}(\partial W, g)}\right)^{\frac{2}{n}} \cdot \frac{Y(\partial W,[g])}{\operatorname{Vol}(\partial W, g)^{\frac{2}{n(n-1)}}} \\
& \leq \frac{n(n-1)}{(n-1)(n-2)} \cdot\left(\frac{\operatorname{Vol}\left(S_{+}^{n}, g_{S^{n}}\right)}{\operatorname{Vol}\left(S^{n-1}, g_{S^{n-1}}\right)}\right)^{\frac{2}{n}} \cdot \frac{Y(\partial W,[g])}{\operatorname{Vol}(\partial W, g)^{\frac{2}{n(n-1)}}} \\
& =\frac{n(n-1) \operatorname{Vol}\left(S_{+}^{n}, g_{\left.S^{n}\right)^{\frac{2}{n}}}\right.}{(n-1)(n-2) \operatorname{Vol}\left(S^{n-1}, g_{S^{n-1}}\right)^{\frac{2}{(n-1)}}} \cdot\left(\frac{\operatorname{Vol}\left(S^{n-1}, g_{S^{n-1}}\right)}{\operatorname{Vol}(\partial W, g)}\right)^{\frac{2}{n(n-1)}} \cdot Y(\partial W,[g]) \\
& =\left(\frac{\operatorname{Vol}\left(S^{n-1}, g_{S^{n-1}}\right)}{\operatorname{Vol}(\partial W, g)}\right)^{\frac{2}{n(n-1)}} \cdot \frac{Y\left(S_{+}^{n}, S^{n-1},\left[g_{S^{n}}\right]\right)}{Y\left(S^{n-1},\left[g_{S^{n-1}}\right]\right)} \cdot Y(\partial W,[g]) .
\end{aligned}
$$

\section{Proof of Theorem 1.1}

Proof of Theorem 1.1 Step 1 Consider the two conformal relative metrics $\bar{g}$ and $\check{g}=\phi^{-2}$. $\bar{g}\left(\phi \in C_{>0}^{\infty}(W)\right)$ given in Theorem 1.1. Then the following formula holds [15] (cf. [17]):

$$
E_{\check{g}}=E_{\bar{g}}+(n-2) \phi^{-1}\left(\bar{\nabla}^{2} \phi-\frac{\Delta_{\bar{g}} \phi}{n} \cdot \bar{g}\right) \text { on } W,
$$

where $E_{\check{g}}:=\operatorname{Ric}_{\check{g}}-\frac{R_{\breve{g}}}{n} \cdot \check{g}$. By the assumption that both $\bar{g}$ and $\check{g}$ are Einstein, we get

$$
\bar{\nabla}^{2} \phi=\frac{\Delta_{\bar{g}} \phi}{n} \cdot \bar{g} \text { on } W .
$$

By the assumption that both $\bar{g}$ and $\check{g}$ are relative metrics, we then have that $\frac{\partial \phi}{\partial t}=0$ along $\partial W$ in terms of Fermi coordinates $(t, x)=\left(t, x^{1}, \cdots, x^{n-1}\right)$ around $\partial W$. From this, we also note that

$$
\bar{\nabla}_{\partial t}^{2} \phi=\partial_{t}^{2} \phi \quad \text { on } U_{\varepsilon}(\partial W), \quad \bar{\nabla}_{\partial_{x^{i}}} \bar{\nabla}_{\partial_{x^{i}}} \phi=\nabla_{\partial_{x^{i}}} \nabla_{\partial_{x^{i}}} \phi, \quad \Delta_{\bar{g}} \phi=\Delta_{g} \phi \quad \text { along } \partial W,
$$

where $\partial_{t}=\frac{\partial}{\partial t}, \partial_{x^{i}}=\frac{\partial}{\partial x^{i}}$ and $\nabla$ denotes the Levi-Civita connection of $g:=\left.\bar{g}\right|_{\partial W}$. Combining the above Hessian equation on $W$ with these equations, we then get

$$
\nabla^{2} \phi=\frac{\Delta_{g} \phi}{n-1} \cdot g \text { on } \partial W
$$

Step 2 Case 2-1 Assume that ( $\partial W,[g])$ is not conformally equivalent to $\left(S^{n-1},\left[g_{S^{n-1}}\right]\right)$. Applying a result of Tashiro [18] (cf. [13, Theorem 21]) to $(\partial W, g)$ with the Hessian equation (7) for $\phi$, we have that, if $\phi \not \equiv$ const $>0$ on $\partial W$, then $(\partial W,[g])$ is conformally equivalent to $\left(S^{n-1},\left[g_{S^{n-1}}\right]\right)$. It contradicts our assumption. Hence, we get that $\phi \equiv$ const $>0$ on $\partial W$. Suppose that $\phi \not \equiv$ const $>0$ on $W$. Then, $\operatorname{Crit}\left(\left.\phi\right|_{\operatorname{Int}(W)}\right) \neq \emptyset$. Take a point $p \in \operatorname{Crit}\left(\left.\phi\right|_{\operatorname{Int}(W)}\right)$ and fix it. By applying another result of Tashiro [19] (cf. [13, Lemmas 13, 18, Corollary 19]) to the two Einstein metrics $\bar{g}$ and $\check{g}=\phi^{-2} \cdot \bar{g}$ with the Hessian equation (6) for $\phi$, there 
exists an open neighborhood $U(\subset \operatorname{Int}(W))$ of $p$ such that $\bar{g}$ is a metric of positive constant curvature on $U$. Then, $\left|W_{\bar{g}}\right|^{2}=0$ on $U$, where $W_{\bar{g}}$ denotes the Weyl curvature tensor of $\bar{g}$. Recall that $(\operatorname{Int}(W), \bar{g})$ is a real analytic Einstein manifold since $\bar{g}$ is Einstein on $W$. Hence $\left|W_{\bar{g}}\right|^{2}=0$ on $\operatorname{Int}(W)$ (and so on $W$ ). Using again that $\bar{g}$ is a positive Einstein metric on $W$, we finally conclude that $\bar{g}$ is a metric of positive constant curvature on $W$, and thus $\check{g}$ is so too.

Case 2-2 Assume that ( $\partial W,[g])$ is conformally equivalent to $\left(S^{n-1},\left[g_{S^{n-1}}\right]\right)$. Suppose that $\phi \not \equiv$ const $>0$ on $W$. If $\operatorname{Crit}\left(\left.\phi\right|_{\operatorname{Int}(W)}\right) \neq \emptyset$, the same argument as Case 2-1 implies that $\bar{g}$ is a metric of positive constant curvature on $W$. Moreover, suppose that $\operatorname{Crit}\left(\left.\phi\right|_{\operatorname{Int}(W)}\right)=\emptyset$. Then, $\operatorname{Crit}\left(\left.\phi\right|_{\partial W}\right) \neq \varnothing$ since $\phi \not \equiv$ const $>0$ on $W$. Take a point $p \in \operatorname{Crit}\left(\left.\phi\right|_{\partial W}\right)$ and fix it. The fact that $\frac{\partial \phi}{\partial t}=0$ along $\partial W$ implies that $p \in \operatorname{Crit}(\phi)$. Take a small open geodesic ball $B$ satisfying $B \subset \operatorname{Int}(W)$ and $\bar{B} \cap \partial W=\{p\}$. One can check that the argument in the result of Tashiro [13, Lemmas 13 and 18, Corollary 19] is still valid for polar coordinates on $(\bar{B}, \bar{g})$ centered at $p \in \partial W \cap \bar{B}$. Then, we have also that $\bar{g}$ is a metric of positive constant curvature on $W$, and thus $\check{g}$ is so too.

In any case, $\phi \equiv$ const $>0$ on $W$ unless $\bar{g}$ is a metric of positive constant curvature on $W$. Hence, this completes the proof of the second assertion (2) in Theorem 1.1.

Step 3 We finally assume that $\bar{g}$ is a metric of positive constant curvature on $W$. This implies that $\check{g}$ is so too. Without loss of generality, we may also assume that both $\bar{g}$ and $\check{g}$ are of constant curvature one. Let $\pi:(\widetilde{W}, \widetilde{g}) \rightarrow(W, \bar{g})$ be the universal Riemannian covering. We claim here that $\partial \widetilde{W}$ is connected even if $\widetilde{W}$ may not be compact. Suppose that $\partial \widetilde{W}$ is not connected. Since the deck transformation group of $\pi$ acts transitively on each fiber $\pi^{-1}(q)(q \in W)$ and that the base space $W$ is compact, one can check that the same argument as the proof of Frankel-Petersen-Wilhelm theorem [16, Theorem 3] is still applicable to our case. Namely, there exist two connected components $V_{1}, V_{2}$ of $\partial \widetilde{W}$ and a minimizing geodesic $\gamma$ joining $V_{1}$ and $V_{2}$. Then, combining the Synge's second variational formula for the length of $\gamma$ with zero mean curvature of $V_{1}, V_{2}$ and the positivity of Ricci curvature of $\tilde{g}$, we can get a contradiction. Hence, $\partial \widetilde{W}$ is connected.

By Cartan-Ambrose-Hicks extension theorem in Riemannian Geometry, there exists an isometric immersion $\delta:(\widetilde{W}, \widetilde{g}) \rightarrow\left(S^{n}, g_{S^{n}}\right)$. Take any $p \in \partial \widetilde{W}$ and fix it. We can also have an isometric immersion $\sigma:(\widetilde{W}, \widehat{g}) \rightarrow\left(S^{n}, g_{S^{n}}\right)$ with $\sigma(p)=\delta(p) \in S^{n}$, where $\widehat{g}:=\pi^{*} \check{g}=(\phi \circ \pi)^{-2} \cdot \widetilde{g}$. Then, there exists an open neighborhood $U$ (in $W$ ) around $p$ such that both $\left.\delta\right|_{U}$ and $\left.\sigma\right|_{U}$ are isometric embeddings. Since $\left(\left.\sigma\right|_{U}\right) \circ\left(\left.\delta\right|_{U}\right)^{-1}:\left(\delta(U), g_{S^{n}}\right) \rightarrow$ $\left(\sigma(U), g_{S^{n}}\right)$ is a conformal diffeomorphism, then there exists $\varphi \in \operatorname{Conf}\left(S^{n},\left[g_{S^{n}}\right]\right)$ such that $\left(\left.\sigma\right|_{U}\right) \circ\left(\left.\delta\right|_{U}\right)^{-1}=\left.\varphi\right|_{\delta(U)}$. This implies that $\varphi^{*} g_{S^{n}}=\left(\phi \circ \pi \circ \delta^{-1}\right)^{-2} \cdot g_{S^{n}}$ on $\delta(U)\left(\subset S^{n}\right)$. Changing $\sigma$ if necessary, we may assume that $S^{n}-(\delta(U) \cup \sigma(U)) \neq \emptyset$. We take a point $p_{\infty} \in S^{n}-(\delta(U) \cup \sigma(U))$. We also assume that $\varphi \in \operatorname{Conf}\left(\mathbb{R}^{n}=S^{n}-\left\{p_{\infty}\right\},\left[g_{\mathbb{E}}\right]\right)$ with $\varphi\left(p_{\infty}\right)=p_{\infty}$, where $g_{\mathbb{E}}$ denotes the Euclidean metric on $\mathbb{R}^{n}$. Then, there exist $\lambda>0$ and $v \in \mathbb{R}^{n}$ such that

$$
\begin{aligned}
\varphi^{*} g_{S^{n}} & =\varphi^{*}\left(\left(\frac{2}{1+|z|^{2}}\right)^{2} \cdot g_{\mathbb{E}}\right), \quad z \in \mathbb{R}^{n} \\
& =\left(\frac{2 \lambda}{\lambda^{2}+|z-v|^{2}}\right)^{2} \cdot g_{\mathbb{E}} \\
& =\left(\frac{\lambda\left(1+|z|^{2}\right)}{\lambda^{2}+|z-v|^{2}}\right)^{2} \cdot g_{S^{n}} .
\end{aligned}
$$


Hence,

$$
\phi \circ \pi \circ \delta^{-1}=\frac{\lambda^{2}+|z-v|^{2}}{\lambda\left(1+|z|^{2}\right)} \quad \text { on } \delta(U)\left(\subset \mathbb{R}^{n}=S^{n}-\left\{p_{\infty}\right\}\right) \text {. }
$$

Note that, if $\lambda=1$ and $v=\mathbf{0}$, then $\varphi$ is an isometry of $\left(S^{n}, g_{S^{n}}\right)$ and that $\phi \equiv 1$ on $\pi(U)(\subset W)$. Since both $\bar{g}$ and $\breve{g}=\phi^{-2} \cdot \bar{g}$ are analytic metrics on $\operatorname{Int}(W)$, then $\phi$ is an analytic function on $\operatorname{Int}(W)$, and hence $\phi \equiv 1$ on $W$. Thus, $\check{g}=\bar{g}$.

Case 3-1 Assume that $(\partial W,[g])$ is not conformally equivalent to $\left(S^{n-1},\left[g_{S^{n-1}}\right]\right)$. By the argument of Case 2-1, we then have that $\phi \equiv 1$ on $\partial W$. Suppose that $\phi \not \equiv 1$ on $W$. This implies that either $\lambda \neq 1$ or $v \neq \mathbf{0}$. Then,

$$
\partial(\delta(U))=\delta(\partial \widetilde{W} \cap U) \subset\left\{z \in \mathbb{R}^{n}\left|\lambda\left(1+|z|^{2}\right)=\lambda^{2}+\right| z-\left.v\right|^{2}\right\},
$$

and hence, for any $z \in \partial(\delta(U))$,

$$
T_{z}\left(\partial(\delta(U))=\left\{w \in \mathbb{R}^{n} \mid\langle w,(\lambda-1) z+v\rangle=0\right\} .\right.
$$

Thus,

$$
T_{z}^{\perp}(\partial(\delta(U))=\{t \cdot((\lambda-1) z+v) \mid t \in \mathbb{R}\} .
$$

Recall that $\frac{\partial \phi}{\partial t}=0$ along $\partial W$ in terms of Fermi coordinates $\left(t, x^{1}, \cdots, x^{n-1}\right)$. We then get that $\operatorname{grad}_{z}\left(\phi \circ \pi \circ \delta^{-1}\right)=-2 \lambda^{-1}\left(1+|z|^{2}\right)^{-1}((\lambda-1) z+v)=0$ for any $z \in \partial(\delta(U))$. This implies that $\lambda=1$ and $v=\mathbf{0}$, and hence this leads to a contradiction. We can now conclude the assertion (1.2) that $\phi \equiv 1$ on $W$ and $\check{g}=\bar{g}$.

Case 3-2 Assume that $(\partial W,[g])$ is conformally equivalent to $\left(S^{n-1},\left[g_{S^{n-1}}\right]\right)$. We consider the restriction $\left.\pi\right|_{\partial \widetilde{W}}:\left(\partial \widetilde{W},\left.\widetilde{g}\right|_{\partial \widetilde{W}}\right) \rightarrow(\partial W, g)$ of the universal Riemannian covering $\pi$ : $(\widetilde{W}, \widetilde{g}) \rightarrow(W, \bar{g})$ to $\partial \widetilde{W}$, which is also a Riemannian covering. Since $\partial \widetilde{W}$ is connected and that $\partial W \cong S^{n-1}$, then the restriction $\left.\pi\right|_{\partial \widetilde{W}}:\left(\partial \widetilde{W},\left.\widetilde{g}\right|_{\partial \widetilde{W}}\right) \rightarrow(\partial W, g)$ must be an isometry. Hence, the covering $\pi$ is a 1-fold Riemannian covering, namely an isometry, and thus $W$ is simply connected. By Cartan-Ambrose-Hicks extension theorem again, there exists an isometric embedding $\delta:(W, \bar{g}) \rightarrow\left(S^{n}, g_{S^{n}}\right)$. By the assumption that $(\partial W,[g])$ is conformally equivalent to $\left(S^{n-1},\left[g_{S^{n-1}}\right]\right)$, there exists a closed geodesic ball $\overline{B_{r}\left(p_{0}\right)}$ of radius $r>0$ centered at $p_{0}$ in $\left(S^{n}, g_{S^{n}}\right)$ such that $\delta(W)=\overline{B_{r}\left(p_{0}\right)}$. If $r \neq \frac{\pi}{2}$, then $\partial \overline{B_{r}\left(p_{0}\right)}$ is not minimal, and so $\partial W$ is not minimal. Hence, $r=\frac{\pi}{2}$, and that $(W, \bar{g})$ is isometric to $\left(S_{+}^{n}, g_{S^{n}}\right)$. It then follows from the assertion (1) of Escobar Theorem that the assertion (1.1) of Theorem 1.1 holds. This completes the proof of Theorem 1.1.

Acknowledgements The author would like to thank Harish Seshadri for valuable comments. He would also like to thank an anonymous referee for helpful comments.

Open Access This article is licensed under a Creative Commons Attribution 4.0 International License, which permits use, sharing, adaptation, distribution and reproduction in any medium or format, as long as you give appropriate credit to the original author(s) and the source, provide a link to the Creative Commons licence, and indicate if changes were made. The images or other third party material in this article are included in the article's Creative Commons licence, unless indicated otherwise in a credit line to the material. If material is not included in the article's Creative Commons licence and your intended use is not permitted by statutory regulation or exceeds the permitted use, you will need to obtain permission directly from the copyright holder. To view a copy of this licence, visit http://creativecommons.org/licenses/by/4.0/.

\section{References}

1. Akutagawa, K., Botvinnik, B.: The relative Yamabe invariant. Comm. Anal. Geom. 13, 935-969 (2002) 
2. Araújo, H.: Critical points of the total scalar curvature plus total mean curvature functional. Indiana Univ. Math. J. 52, 85-107 (2003)

3. Besse, A.: Einstein Manifolds. Springer, New York (1987)

4. Brendle, S., Chen, S.: An existence theorem for the Yamabe problem on manifolds with boundary. J. Eur. Math. Soc. 16, 991-1016 (2014)

5. Cherrier, P.: Problèmes de Neumann non liéaires sur les variétés riemanniennes. J. Funct. Anal. 57, 154-206 (1984)

6. DeTurck, D., Kazdan, J.: Some regularity theorem in Riemannian Geometry. Ann. Scient. Ec. Norm. Sup. $4^{\circ}$ serie 14, 249-260 (1981)

7. Escobar, J.F.: Uniqueness theorems on conformal deformatio of metrics, Sobolev inequalities, and an eigenvalue estimate. Comm. Pure Appl. Math. 43, 857-883 (1990)

8. Escobar, J.F.: The Yamabe problem on manifolds with boundary. J. Differ. Geom. 35, 21-84 (1992)

9. Frankel, T.: Manifolds with positive curvature. Pac. J. Math. 11, 165-174 (1961)

10. Gromov, M.: Paul Levy's isoperimetric inequality, preprint, IHES/M/80/320 (1980)

11. Gilbarg, D., Trudinger, N.S.: Elliptic Partial Differential Equations of Second Order, Springer, Grundlehren 224. Heidelberg, New York, Berlin (1977)

12. Gursky, M., Han, Q.: Non-existence of Poncaré-Einstein manifolds with prescribed conformal infinity. Geom. Funct. Anal. 27, 863-879 (2017)

13. Kühnel, W.: Conformal transformations between Einstein spaces, Conformal Geometry (R. S. Kulkarni and U. Pinkall Eds.), 105-146. Aspects of Mathematics: E, 1, Vieweg (1988)

14. Obata, M.: Certain conditions for a Riemannian manifold to be isometric with a sphere. J. Math. Soc. Jpn. 14, 333-340 (1962)

15. Obata, M.: The conjectures on conformal transformations of Riemannian manifolds. J. Differ. Geom. 6, 247-258 (1971)

16. Petersen, P., Wilhelm, F.: On Frankel's Theorem. Canad. Math. Bull. 46, 130-139 (2003)

17. Schoen, R.: Variational theory for the total scalar curvature functional for Riemannian metrics and related topics, Topics in calculus of variations (Montecatini Terme, 1987), 120-154, Lecture Notes in Math. 1365, Springer-Verlag (1989)

18. Tashiro, Y.: Complete Riemannian manifolds and some vector fields, Trans. Am. Math. Soc. 117, 251-275 (1957)

19. Tashiro, Y.: Conformal transformations in complete Riemannian manifolds. Publ. Group of Geometry 3 (1967)

Publisher's Note Springer Nature remains neutral with regard to jurisdictional claims in published maps and institutional affiliations. 